\title{
Dexamethasone Implant Migration through an Iris Coloboma
}

\author{
Yoav Glidai ${ }^{a, b} \quad$ Shulamit Schwartz ${ }^{a, b} \quad$ Eyal Cohen ${ }^{a, b}$ \\ aDepartment of Ophthalmology, Tel Aviv Medical Center, Tel Aviv, Israel; bSackler School \\ of Medicine, Tel Aviv University, Tel Aviv, Israel
}

\section{Keywords}

Ozurdex · Implant $\cdot$ Migration · Iris coloboma $\cdot$ Surgical technique

\begin{abstract}
Ozurdex ${ }^{\circledR} 0.7$ mg (dexamethasone 0.7 mg implant, Allergan, Dublin, Ireland), an intravitreal biodegradable implant, is indicated for cystoid macular edema due to various causes. One of its known and uncommon complications is implant migration to the anterior chamber, causing corneal edema that, in some cases, is irreversible. Reported risk factors for device migration are open or defective lens capsule and prior history of vitrectomy. We present a case of dexamethasone implant migration through a congenital iris coloboma in a pseudophakic patient with an intact lens capsule. The patient is a 56-year-old pseudophakic man with a history of congenital iris coloboma, myopia, retinal tears, and a branch retinal vein occlusion with subsequent cystoid macular edema resistant to anti-VEGF medications but responsive to corticosteroids. He presented with sudden painless decreased vision in his left eye, 8 weeks following dexamethasone implant (Ozurdex) injection to the same eye. Upon presentation, he was diagnosed with corneal edema caused by anterior chamber migration of the implant. He was referred for immediate surgical intervention to extract the implant, with a resolution of the corneal edema within 2 weeks postoperatively. To conclude, this is the first case that reports Ozurdex implant migration through an iris coloboma in the setting of an intact posterior capsule. In addition, we describe a novel surgical approach for implant removal from the anterior chamber that is simple and efficient.




\section{Case Reports in Ophthalmology}

\section{Background}

Ozurdex ${ }^{\circledR} 0.7 \mathrm{mg}$ (dexamethasone $0.7 \mathrm{mg}$ implant, Allergan, Dublin, Ireland) is a 0.46 by $6 \mathrm{~mm}$ biodegradable implant, injected into the vitreous cavity for the treatment of macular edema due to several conditions, among them cystoid macular edema (CME) due to vein occlusion [1]. In recent years, clinical indications for this treatment have been expanded, and episodes of implant migration to the anterior chamber (AC) have been reported. The main risk factors for device migration are open or defective lens capsule and prior history of vitrectomy [2, 3]. Recently, Stepanov et al. [4] and Stelton et al. [5] reported of implant migration in patients with an iris defect, suggesting it could serve as the migration route. Of note, all the reported cases had, in addition to an iris defect, a defective lens capsule. To the best of our knowledge, there has been only one report of migration in the setting of an intact posterior capsule, in which migration was speculated to occur through weak or ruptured zonules [6].

When an Ozurdex device migrates to the AC, it can cause rapid development of corneal edema and as a consequence, corneal decompensation [2,3], therefore it is crucial to remove the implant promptly. We present a case of dexamethasone implant migration through a congenital iris coloboma in a pseudophakic patient with an intact lens capsule.

\section{Case Presentation}

A 56-year-old pseudophakic man presented with a sudden painless decrease in vision in his left eye (LE), 8 weeks following dexamethasone implant (Ozurdex) injection to the same eye. He was treated with Ozurdex implant for macular edema resulting from branch retinal vein occlusion (BRVO) that occurred 4 years prior to his current presentation; this was his second Ozurdex implantation. His other past ocular history included congenital inferior iris coloboma in his LE, axial myopia in both eyes, right $11 \mathrm{dpt}$, and left $2 \mathrm{dpt}$. His myopia was corrected at a young age with glasses, and amblyopia was not documented at any stage. The patient underwent laser refractive surgery correction in his right eye (RE) when reaching adulthood. Additionally, he had posterior vitreous detachment (PVD) in both eyes and a history of several retinal tears in both eyes treated with laser retinopexy and cryotherapy. Three years prior to his presentation, the patient had uneventful cataract surgery to his LE with posterior chamber intraocular lens (PCIOL) implantation.

The patient had experienced BRVO in his LE with subsequent macular edema 4 years before his presentation. When he began retinal clinic follow-up and treatment at our center, his best-corrected visual acuity (BCVA) was $6 / 6$ and $1 / 24$ in his RE and LE, respectively. A macular optical coherence tomography (OCT) of his LE demonstrated CME with an elevated central macular thickness (CMT) of $465 \mu \mathrm{m}$. During his retinal clinic follow-up, he was treated with intravitreal anti-VEGF agents, including bevacizumab, ranibizumab, and aflibercept injections, showing no improvement of the macular edema and even worsening, with LE VA decreasing to $1 / 36$. As second-line therapy, triamcinolone was injected intravitreally, resulting in partial improvement of the macular edema with a fair amount of fluid absorption. Macular OCT under triamcinolone treatment demonstrated a decrease in CMT from 507 to $440 \mu \mathrm{m}$. As this improvement suggested a good response to corticosteroid treatment, he qualified for Ozurdex implant injection. The first implant was inserted with no complications. One month following the insertion, we witnessed a significant improvement in his LE macular edema, with an OCT scan showing a decrease in measured CMT from 405 to $311 \mu \mathrm{m}$ and BCVA improving from 
$1 / 36$ to $6 / 60$. Eight weeks before his presentation, he underwent a second Ozurdex injection with a similar response.

The patient presented with sudden decreased "hazy" vision in his LE; visual acuity was $1 / 36$ in his LE. Slit-lamp examination revealed central and inferior stromal corneal edema in his LE. The AC was deep, with the dexamethasone implant lying in the inferior angle touching the inferior cornea (Fig. 1). Iris coloboma was open, and PCIOL was correctly situated in the bag with an intact posterior capsule, LE intraocular pressure measured $18 \mathrm{~mm} \mathrm{Hg}$.

After ruling out other causes for corneal edema, we estimated that the etiology was implant migration. A literature review on such cases was conducted, concluding that prompt extraction of the implant is required to avoid persistent corneal decompensation. The patient was referred for surgical intervention within $24 \mathrm{~h}$ from his presentation.

The surgery was initiated by making two $1.0-\mathrm{mm}$ corneal incisions 180 degrees apart, introducing an AC maintainer with a basic salt solution through one incision and a spatula gently opening the second incision directly across. Due to the pressure gradient and stream created by the maintainer, the dexamethasone implant was navigated toward the second incision and easily ejected from the AC. Following implant extraction, intracameral cefuroxime was injected, and incision hydration was made (online suppl. Video; for all online suppl. material, see www.karger.com/doi/10.1159/000505638).

One day postoperatively on eye examination, the corneal edema was still evident, AC was deep and quiet, intraocular pressure was $15 \mathrm{~mm} \mathrm{Hg}$. On examination 2 weeks postoperatively, the patients' LE BCVA was 6/120, and corneal edema had resolved entirely. Corneal endothelial cell density measured 903 cells $/ \mathrm{mm}^{2}$ in his LE and 2,474 cells $/ \mathrm{mm}^{2}$ in his RE, macular OCT showed increased macular edema with CMT rising from 342 to $477 \mu \mathrm{m}$. Following careful deliberation (elaborated in "Discussion"), we found that the best course of action would be a repeated injection of Ozurdex, and the patient underwent a third implant injection to his LE. In follow-up visits, there has not been a recurrence of implant migration.

\section{Discussion}

Anterior chamber Ozurdex implant migration is a rare complication that can lead to permanent corneal decompensation. In a retrospective study, Khurana et al. [3] showed that early removal of the implant is essential for corneal recovery. The "classic" risk factors for implant migration identified in that study were a history of pars plana vitrectomy and open or defective lens capsule. In a recent review article by Rahimy and Khurana [2], two additional risk factors were described: iris defect (patent laser iridotomy or surgical iridectomy) and zonular dehiscence (from previous trauma or cataract surgery). Kocak et al. [6] were the first to describe implant migration in the setting of an intact posterior capsule, assuming the culprits were weak zonules. Two cases have been reported about iris defects being the route of passage. First was Stelton et al. [5], who described a patient with PCIOL and a disrupted posterior capsule that also had a large peripheral inferior iridectomy. Later, Stepanov et al. [4] described a patient that underwent inferior iridectomy due to silicone oil insertion during pars plana vitrectomy for treating retinal detachment, which was later on implanted with iris-claw ACIOL (Artisan). Although it is fairly likely that in both cases the implant migrated through the iris defects, both patients also had the "classic" risk factors (prior pars plana vitrectomy and disrupted lens capsule). In the presented case, the patient had an intact posterior capsule. As discussed by Stepanov et al. [4], an iris defect that is situated in the inferior iris (as in our case) 
is more susceptible to be a route of passage for an implant, as it usually sinks to the bottom of the vitreous cavity.

Congenital iris colobomas arise from incomplete closure of the embryonic fissure during fetal development. A "typical" congenital iris coloboma is located at the inferonasal quadrant and, therefore, must be considered a risk factor for an AC implant migration. This case is the first report of Ozurdex implant migration through an iris coloboma in the absence of capsular defects. It is reasonable to assume that the migration occurred through weak, absent or defective zonules, perhaps as a result of his previous cataract surgery or a congenital defect that is associated with the iris coloboma. The presence of PVD in this scenario is also likely to have facilitated the dislocation and mobility of the implant. Therefore, it is essential to inform these patients of having an increased risk for implant migration and the importance of seeking an immediate medical consultation with any sudden change of vision as early removal of the implant increases corneal recovery rates [3].

Following the migrating implant removal, the patient had an exacerbation of the CME. Our team of experts came together for a convergent thinking process, with several alternatives weighed. The first option considered was to reinitiate injections of anti-VEGF agents. However, our patient was unresponsive to all anti-VEGF treatments; therefore, a repeated trial would probably be infertile. The second alternative considered was implanting a different intraocular steroidal implant. Fluocinolone acetonide $0.59 \mathrm{mg}$ (Retisert ${ }^{\circledR}$, Bausch and Lomb, Rochester, NY, USA) and fluocinolone acetonide $0.19 \mathrm{mg}$ (Iluvien ${ }^{\circledR}$, Alimera Sciences, $\mathrm{Al}$ pharetta, GA, USA) are two steroidal implants available for intraocular use. Although Iluvien is designed to provide sustained therapy for a more extended period, it is also free-floating, but smaller in size than Ozurdex, making it even more susceptible to migrate. Only two cases of Iluvien migration have been reported, with both patients suffering from consequent corneal edema [7, 8]. Despite being anchored to the sclera, Retisert has also been reported to dislocate to the $\mathrm{AC}$, inducing corneal edema, possibly as a result of medication reservoir dissociation from its sutured strut [9-11].

Another possibility considered was iris coloboma repair, pupilloplasty. Despite being an appealing alternative, pupilloplasty is an additional anterior segment surgery, which involves a risk to a cornea that has already gone through a considerable amount of procedures, thus jeopardizing it again. A corneal endothelial cell density of $903 / \mu \mathrm{m}^{2}$ in his $\mathrm{LE}$ further reinforced the impression that additional surgery should be avoided. Moreover, the patients' extensive ocular history was taken into consideration. The patient LE BCVA was only slightly improved from $1 / 36$ to 20/200 during Ozurdex treatment, despite the absorption of the macular edema. We estimated that the visual potential in the LE is limited due to the extended presence of subretinal fluid and photoreceptor compromise. Since Ozurdex treatment had a substantial effect on the patients' CME and improved his VA, repeating the procedure was positively considered. After thorough deliberation on the above alternatives, we decided to repeat Ozurdex insertion, concluding that any other option would carry either the same or a higher risk to the patients' eye. The patient accepted our recommendation and underwent a third Ozurdex implantation. The patient was instructed to return at any sudden change of vision. As mentioned, in a follow-up of 12 months and repeated Ozurdex injections, implant migration did not recur.

\section{Conclusion}

We have presented a case of dexamethasone implant migration to the AC through a congenital coloboma, resulting in corneal edema. This report is the first description of implant 
migration through a congenital iris coloboma in the setting of an intact posterior capsule. We also described our surgical approach for implant removal that is simple, efficient, and costeffective. Physicians should inform patients with iris defects of the risk of implant migration. It is eminent to emphasize that at any decrease or change in vision, the patient should seek urgent medical attention.

\section{Acknowledgement}

No organization has contributed to this or the manuscript.

\section{Statement of Ethics}

Written informed consent was obtained from the patient for publication of this article and any accompanying images. A copy of the written consent is available for review by the editor of this journal.

\section{Disclosure Statement}

The authors have no conflicts of interest to declare.

\section{Funding Sources}

No financial support was received for this submission.

\section{Authors Contribution}

Y.G. contributed to the manuscript conception, design, and acquisition. E.C. and S.S. contributed to the acquisition, analysis, and interpretation of data. All authors have been involved in drafting the manuscript or revising it critically and have given final approval of this version to be published and agreed to be accountable for all aspects of the work.

\section{References}

1 Haller JA, Bandello F, Belfort R Jr, Blumenkranz MS, Gillies M, Heier J, et al.; OZURDEX GENEVA Study Group. Randomized, sham-controlled trial of dexamethasone intravitreal implant in patients with macular edema due to retinal vein occlusion. Ophthalmology. 2010 Jun;117(6):1134-1146.e3.

2 Rahimy E, Khurana RN. Anterior segment migration of dexamethasone implant: risk factors, complications, and management. Curr Opin Ophthalmol. 2017 May;28(3):246-51.

3 Khurana RN, Appa SN, McCannel CA, Elman MJ, Wittenberg SE, Parks DJ, et al. Dexamethasone implant anterior chamber migration: risk factors, complications, and management strategies. Ophthalmology. 2014 Jan;121(1):67-71.

4 Stepanov A, Codenotti M, Ramoni A, Prati M, Jiraskova N, Rozsival P, et al. Anterior chamber migration of dexamethasone intravitreal implant (Ozurdex®) through basal iridectomy (Ando) in a pseudophakic patient. Eur J Ophthalmol. 2016 Apr;26(3):e52-4. 


\section{Case Reports in Ophthalmology}

(c) 2020 The Author(s). Published by S. Karger AG, Basel www.karger.com/cop

Glidai et al.: Dexamethasone Implant Migration through an Iris Coloboma

5 Stelton CR, Townsend J, Peterson LT, Khurana RN, Yeh S. Surgical Management of Anterior Chamber Migration of a Dexamethasone Intravitreal Implant. Ophthalmic Surg Lasers Imaging Retina. 2015 JulAug;46(7):756-9.

6 Kocak N, Ozturk T, Karahan E, Kaynak S. Anterior migration of dexamethasone implant in a pseudophakic patient with intact posterior capsule. Indian J Ophthalmol. 2014 Nov;62(11):1086-8.

7 Papastavrou VT, Zambarakji H, Dooley I, Eleftheriadis H, Jackson TL. OBSERVATION: FLUOCINOLONE ACETONIDE (ILUVIEN) IMPLANT MIGRATION INTO THE ANTERIOR CHAMBER. Retin Cases Brief Rep. 2017;11(1):44-6.

8 El-Ghrably IA, Saad A, Dinah C. A Novel Technique for Repositioning of a Migrated ILUVIEN(®) (Fluocinolone Acetonide) Implant into the Anterior Chamber. Ophthalmol Ther. 2015 Dec;4(2):129-33.

9 Almeida DR, Chin EK, Mears K, Russell SR, Mahajan VB. Spontaneous dislocation of a fluocinolone acetonide implant (Retisert) into the anterior chamber and its successful extraction in sympathetic ophthalmia. Retin Cases Brief Rep. 2015;9(2):142-4.

10 Chang PY, Kresch Z, Samson CM, Gentile RC. Spontaneous Dissociation of Fluocinolone Acetonide Sustained Release Implant (Retisert) with Dislocation into the Anterior Chamber. Ocul Immunol Inflamm. 2015;23(6):454-7.

11 Akduman L, Cetin EN, Levy J, Becker MD, Mackensen F, Lim LL. Spontaneous dissociation and dislocation of Retisert pellet. Ocul Immunol Inflamm. 2013;21(1):87-9.

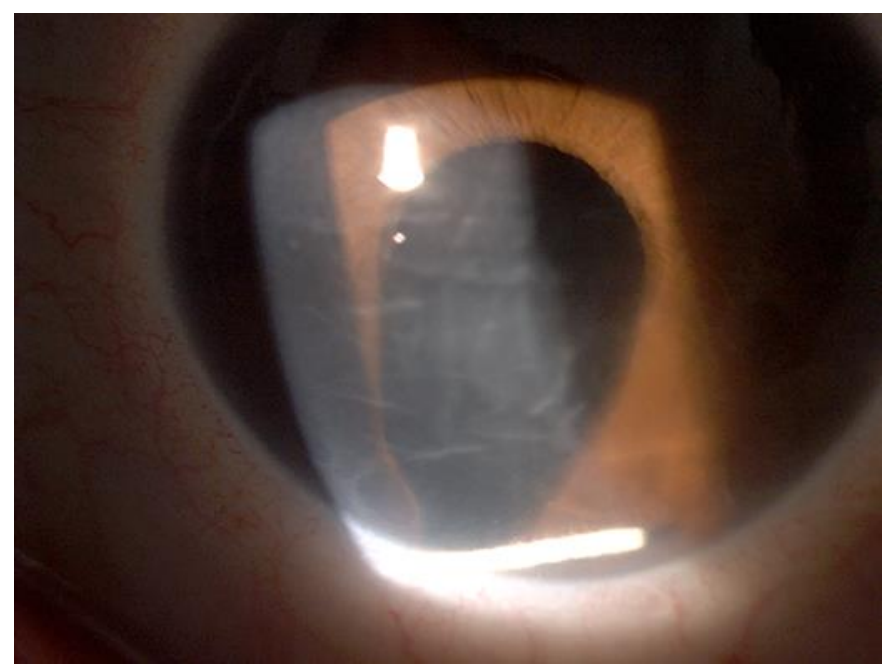

Fig. 1. Slit-lamp biomicroscopy capturing dexamethasone implant dislocated through inferior iris coloboma to the anterior chamber with corneal edema and Descemet folds. 